\title{
Relating quotient completions via categorical logic
}

\author{
Maria Emilia Maietti* Giuseppe Rosolini ${ }^{\dagger}$
}

16th June 2016

\begin{abstract}
In previous work we introduced the notion of elementary quotient completion with respect to an elementary doctrine. We also generalized the notion of exact completion of a regular category as an exact completion of an existential elementary doctrine.

Here we characterize when the elementary quotient completion of an elementary existential doctrine coincides with an exact completion.

We do this by employing the categorical logic of the various notions of doctrines involved in our analysis. The outcome is that the two completions coincide when a choice rule holds in the starting existential elementary doctrine.
\end{abstract}

MSC 2000: 03G30 03B15 18C50 03B20 03F55

Keywords: quotient completion, split fibration, universal construction

\section{Introduction}

The need of completing a structure with quotients arises in various fields in mathematics and in computer science, including foundation of mathematics, type theory and category theory, in order to get models closed under quotients though based on much weaker structures.

In previous work [Maietti and Rosolini, 2013b,a] we used the language of category theory to introduce a notion of quotient completion for Lawvere's elementary doctrines, see [Lawvere, 1969a, 1970]. The notion includes well-known instances of quotient completion in category theory and in type theory: for examples, the exact completion of a lex category as in [Carboni and Celia Magno, 1982], Joyal's arithmetic universes (see [Maietti, 2010]) and setoid models of type theory are all instances of elementary quotient completions.

* Dipartimento di Matematica Pura ed Applicata, Università di Padova, via Trieste 63, 35121 Padova, Italy, email: maietti@math.unipd.it

†DIMA, Università di Genova, via Dodecaneso 35, 16146 Genova, Italy, email: rosolini@unige.it

Project Correctness by Construction (EU 7th framework programme, grant no. PIRSES-GA2013-612638) provided support for the research presented in the paper. 
In another work [Maietti and Rosolini, 2015] we reviewed and generalized the notion of exact completion of a regular category as a free construction from an existential elementary doctrine. Examples of this construction are toposes obtained from triposes, see [Hyland et al., 1980], in particular the effective topos of [Hyland, 1982].

The aim of this paper is to present a logical characterization of when the elementary quotient completion of an existential elementary doctrine happens to coincide with its exact completion.

To this purpose in the first part of the paper we review the mentioned notions of quotient completion related to an elementary doctrine. Differently from the previous work, here we employ the internal logic for elementary doctrine as presented in [Jacobs, 1999]. Finally we show that the two completions coincide when the starting elementary existential doctrine satisfies a rule of choice.

\section{Notions of elementary doctrine}

In previous work [Maietti and Rosolini, 2013b,a] we employed the notion of elementary doctrine to generalize the completion of a categorical structure with quotients. The main idea was to relativize the concept of quotient completion to a many sorted logic, represented categorically by a doctrine, validating the logical structure needed to speak of equivalence relations. In loc.cit. we found appropriate to use Lawvere's concept of elementary doctrine, which represents a many sorted logic with conjunctions and propositional equality, and related to it we defined the notion of elementary quotient completion.

F.W. Lawvere introduced the notion of elementary doctrine in a series of seminal papers, see [Lawvere, 1969a,b, 1970], to synthetize the structural properties of logical systems, see also [Lawvere and Rosebrugh, 2003] for a unified survey. Lawvere's crucial intuition was to consider logical languages and theories as hyperdoctrines to study their 2-categorical properties, e.g. connectives and quantifiers are determined by structural adjunctions. That approach proved extremely fruitful, see [Makkai and Reyes, 1977, Carboni, 1982, Lambek and Scott, 1986, Jacobs, 1999, Taylor, 1999, van Oosten, 2008] and references therein.

Taking advantage of the category-theoretical presentation of logic by doctrines, we now first introduce a general notion of elementary doctrine which we found appropriate to study the notion of quotient of an equivalence relation, see [Maietti and Rosolini, 2013b, 2015].

Since doctrines can be presented equivalently in the form of fibrations, see e.g. [Jacobs, 1999], we may use the names interchangeably.

Denote by InfSL the 2-category of inf-semilattice: objects are posets with finite meets, 1-morphisms are functions between them which preserves finite meets, and 2-morphisms are given by the pointwise order on the homsets.

Definition 2.1. Let $C$ be a category with finite products. An elementary doctrine on $C$ is a functor $P: C^{\mathrm{op}} \longrightarrow \operatorname{InfSL}^{1}$ - the value $P(A)$ is the fibre on

\footnotetext{
${ }^{1}$ We may refer to such a functor as an indexed inf-semilattice; we always write $P_{f}$ for the
} 
$A$ - such that, for every object $A$ in $\mathcal{C}$, there is an object $\delta_{A}$, which we shall call fibered equality - in $P(A \times A)$ such that

(*) for every object $X$ and morphism $e:=\left\langle\mathrm{pr}_{1}, \mathrm{pr}_{2}, \mathrm{pr}_{2}\right\rangle: X \times A \rightarrow X \times A \times A$ in $\mathcal{C}$, the assignment that sends $\alpha$ in $P(X \times A)$ to the meet

$$
\oiint_{e}(\alpha):=P_{\mathrm{id}_{X} \times \mathrm{pr}_{1}}(\alpha) \wedge P_{\left\langle\mathrm{pr}_{2}, \mathrm{pr}_{3}\right\rangle}\left(\delta_{A}\right),
$$

computed in $P(X \times A \times A)$, determines a left adjoint to $P_{e}: P(X \times A \times A) \rightarrow$ $P(X \times A)$ where $\operatorname{pr}_{i}, i=1,2$, are the projections from $X \times A$ to each of the two factors.

Remark 2.2. (a) Since $T$ is terminal in $\mathcal{C}$, condition $(*)$ for $X=T$ yields that the assignment that sends $\alpha$ in $P(A)$ to the meet

$$
\mathbb{B}_{\left\langle\mathrm{id}_{A}, \mathrm{id}_{A}\right\rangle}(\alpha):=P_{\mathrm{pr}_{1}}(\alpha) \wedge \delta_{A},
$$

computed in $P(A \times A)$, determines a left adjoint to $P_{\left\langle\mathrm{id}_{A}, \mathrm{id}_{A}\right\rangle}: P(A \times A) \rightarrow$ $P(A)$.

(b) The previous remark yields that $\delta_{A}$ is unique for each object $A$ in $\mathcal{C}$.

(c) Since $\left\langle\operatorname{pr}_{2}, \operatorname{pr}_{1}\right\rangle \circ\left\langle\operatorname{id}_{A}, \operatorname{id}_{A}\right\rangle=\left\langle\operatorname{id}_{A}, \operatorname{id}_{A}\right\rangle$, one has that

$$
\mathbb{H}_{\left\langle\mathrm{id}_{A}, \mathrm{id}_{A}\right\rangle}(\alpha)=P_{\mathrm{pr}_{2}}(\alpha) \wedge \delta_{A}
$$

for every $\alpha$ in $P(A)$.

(d) In $P(A \times A)$ it is $\top \leq P_{\left\langle\operatorname{id}_{A}, \operatorname{id}_{A}\right\rangle}\left(\delta_{A}\right)$ and, in $P(A \times A)$, it is $\delta_{A} \leq P_{f \times f}\left(\delta_{B}\right)$ when $f: A \rightarrow B$.

(e) For $\alpha_{1}$ in $P\left(X_{1} \times Y_{1}\right)$ and $\alpha_{2}$ in $P\left(X_{2} \times Y_{2}\right)$, write $\alpha_{1} \otimes \alpha_{2}$ for the object

$$
P_{\left\langle\mathrm{pr}_{1}, \mathrm{pr}_{3}\right\rangle}\left(\alpha_{1}\right) \wedge P_{\left\langle\mathrm{pr}_{2}, \mathrm{pr}_{4}\right\rangle}\left(\alpha_{2}\right)
$$

in $P\left(X_{1} \times X_{2} \times Y_{1} \times Y_{2}\right)$ where $\operatorname{pr}_{i}, i=1,2,3,4$, are the projections from $X_{1} \times X_{2} \times Y_{1} \times Y_{2}$ to each of the four factors.

Condition 2.1(b) is to request that $\delta_{A \times B}=\delta_{A} \otimes \delta_{B}$ for every pair of objects $A$ and $B$ in $C$.

Examples 2.3. (a) The first example of elementary doctrine is that of powersets. The domain category is the category Set of sets and functions. The terminal object can be fixed as $\{\emptyset\}$, and the product of two sets is given by the set of ordered pairs encoded as usual $(x, y):=\{\{x\},\{x, y\}\}$ with obvious projections.

The elementary doctrine $\mathbf{P}: \operatorname{Set}^{\mathrm{op}} \longrightarrow \mathbf{I n f S L}$ on $\operatorname{Set}$ is given as the powerset $\mathbf{P}(A)$. The action on functions $f: A \rightarrow B$ is by inverse image, i.e. $\mathbf{P}_{f}:=$ $f^{-1}: \mathbf{P}(B) \rightarrow \mathbf{P}(A)$.

The elementary structure is given by the diagonal subset of $A \times A$.

value of the indexing functor $P$ on a morphism $f$. 
(b) The standard categorical example of an indexed poset is the fibration of subobjects, a generalization of the previous example to an arbitrary category. Consider a category $\mathcal{C}$ with binary products, a terminal object and pullbacks of monomorphisms - for convenience, we shall assume that $C$ is also well-powered, here as well as any other time when size issues become relevant. The functor $S: C^{\mathrm{op}} \longrightarrow$ InfSL assigns to any object $A$ in $C$ the poset $S(A)$ of subobjects of $A$ in $C$ and it is easy to check that the poset is an inf-semilattice. For a morphism $f: B \rightarrow A$, the assignment that maps a subobject in $S(A)$ to that represented by the left-hand morphism in any pullback of it along $f$ produces a functor $S_{f}: S(A) \rightarrow S(B)$ that preserves products.

The elementary structure is provided by the diagonal morphisms.

(c) Another categorical example is given by a category $\mathcal{S}$ with binary products and weak pullbacks, by defining the doctrine functor of weak subobjects $\Psi: S^{\mathrm{op}} \longrightarrow$ InfSL which evaluates as the poset reflection of each comma category $\mathcal{S} / A$ at each object $A$ of $\mathcal{S}$, introduced in [Grandis, 2000].

(d) An example of elementary doctrine from first order logic is the LindenbaumTarski algebras of well-formed formulas of a theory $\mathcal{T}$ with equality in the first order language $\mathcal{L}$. The domain category is the category $\mathcal{V}$ of lists of variables and term substitutions:

object of $\mathcal{V}$ are lists of distinct variables $\left(x_{j_{1}}, \ldots, x_{j_{n}}\right)$;

morphisms $\left(t_{1} / x_{k_{1}}, \ldots, t_{m} / x_{k_{m}}\right):\left(x_{j_{1}}, \ldots, x_{j_{n}}\right) \rightarrow\left(x_{k_{1}}, \ldots, x_{k_{m}}\right)$ are lists of substitutions for variables where each term $t_{j}, i=1, \ldots, m$, is built in $\mathcal{L}$ on the variables $x_{j_{1}}, \ldots, x_{j_{n}}$

composition $\left(x_{j_{1}}, \ldots, x_{j_{n}}\right) \stackrel{\left(t_{i} / x_{k_{i}}\right)_{i=1}^{m}}{\longrightarrow}\left(x_{k_{1}}, \ldots, x_{k_{m}}\right) \stackrel{\left(s_{i} / x_{h_{i}}\right)_{i=1}^{\ell}}{\longrightarrow}\left(x_{h_{1}}, \ldots, x_{h_{\ell}}\right)$ is given by simultaneous substitutions

$$
\left(x_{j_{1}}, \ldots, x_{j_{n}}\right) \stackrel{\left.\left(s_{i}\left(t_{1} / x_{k_{1}}, \ldots, t_{m} / x_{k_{m}}\right) / x_{h_{i}}\right)_{i=1}^{\ell}\right)}{\longrightarrow}\left(x_{h_{1}}, \ldots, x_{h_{\ell}}\right) .
$$

The terminal object is the empty list (). The product of two objects $\left(x_{j_{1}}, \ldots, x_{j_{n}}\right)$ and $\left(x_{k_{1}}, \ldots, x_{k_{m}}\right)$ is given by the list $\left(x_{j_{1}}, \ldots, x_{j_{n}}, x_{J+k_{1}}, \ldots, x_{J+k_{m}}\right)$ where $J=\max \left\{j_{1}, \ldots, j_{n}\right\}$. Projections are given by substitution of the variables in $\left(x_{j_{1}}, \ldots, x_{j_{n}}\right)$ with the first $n$ variables in $\left(x_{j_{1}}, \ldots, x_{j_{n}}, x_{J+k_{1}}, \ldots, x_{J+k_{m}}\right)$ and of the variables in $\left(x_{k_{1}}, \ldots, x_{k_{m}}\right)$ with the last $m$ in $\left(x_{j_{1}}, \ldots, x_{j_{n}}, x_{J+k_{1}}, \ldots, x_{J+k_{m}}\right)$. The elementary doctrine $L T: \mathcal{V}^{\mathrm{op}} \longrightarrow \operatorname{InfSL}$ on $\mathcal{V}$ is given as follows: for a list of distinct variables $\vec{x}$, the inf-semilattice $L T(\vec{x})$ has

objects are equivalence classes of well-formed formulas of $\mathcal{L}$ with no more free variables than $x_{j_{1}}, \ldots, x_{j_{n}}$ with respect to provable reciprocal consequence $W \vdash_{\mathcal{T}} W^{\prime}$ in $\mathcal{T}$;

morphisms $[W] \rightarrow[V]$ are the provable consequences $W \vdash_{\mathcal{T}} V$ in $\mathcal{T}$ for some pair of representatives (hence for any pair); 
composition is given by the cut rule in the logical calculus; identities $[W] \rightarrow[W]$ are given by the logical rules $W \vdash_{\mathcal{T}} W$.

For a list of distinct variables $\vec{x}:=\left(x_{j_{1}}, \ldots, x_{j_{n}}\right)$, the poset $L T(\vec{x})$ has finite meets: the top element is any true formula, for example $x=x$ with $x$ variable in $\vec{x}$ and the meet of a pair of formulas is obtained by conjunction.

Elementary doctrines are the cloven Eq-fibrations of [Jacobs, 1999] and, as explained in loc.cit., there is a deductive calculus associated to those which is that of the $\wedge=$-fragment over type theory with just a unit type and a binary product type constructor. From now on, we shall employ the logical language introduced in loc.cit. and write

$$
a_{1}: A_{1}, \ldots, a_{k}: A_{k} \mid \phi_{1}\left(a_{1}, \ldots, a_{k}\right), \ldots, \phi_{n}\left(a_{1}, \ldots, a_{k}\right) \vdash \psi\left(a_{1}, \ldots, a_{k}\right)
$$

in place of

$$
\phi_{1} \wedge \ldots \wedge \phi_{n} \leq \psi
$$

in $P\left(A_{1} \times \ldots \times A_{k}\right)$. Note that, in line with loc.cit., $\delta_{A}\left(a, a^{\prime}\right)$ will be written as $a: A, a^{\prime}: A \mid a={ }_{A} a^{\prime}$. Also we write $a: A \mid \alpha \dashv \vdash \beta$ to abbreviate $a: A \mid \alpha \vdash \beta$ and $a: A \mid \beta \vdash \alpha$.

It is possible to express precisely relationships between the examples once one considers the 2-category ED of elementary doctrines:

the 1-morphisms are pairs $(F, \mathfrak{b})$ where $F: \mathcal{C} \rightarrow \mathcal{D}$ is a functor and $\mathfrak{b}: P \rightarrow$ $R \circ F^{\mathrm{op}}$ is a natural transformation as in the diagram

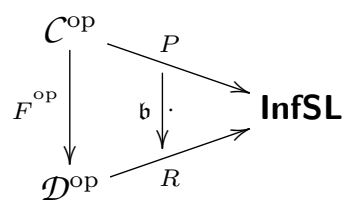

where the functor $F$ preserves products and, for every object $A$ in $C$, the functor $\mathfrak{b}_{A}: P(A) \rightarrow R(F(A))$ preserves finite meets and $\mathfrak{b}_{A \times A}\left(\delta_{A}\right)=$ $R_{\left\langle F\left(\mathrm{pr}_{1}\right), F\left(\mathrm{pr}_{2}\right)\right\rangle}\left(\delta_{F(A)}\right) ;$

the 2-morphisms are natural transformations $\theta: F \rightarrow G$ such that

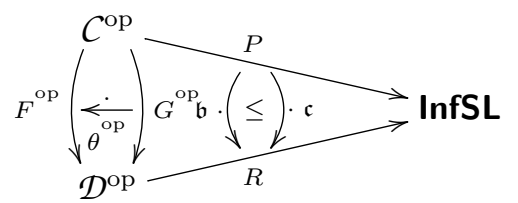

so that, for every $A$ in $\mathcal{C}$ and every $\alpha$ in $P(A)$, one has $\mathfrak{b}_{A}(\alpha) \leq_{F(A)}$ $R_{\theta_{A}}\left(\mathfrak{c}_{A}(\alpha)\right)$. 
Remark 2.4. Expressing in the internal logic the action of a 1-morphism $(F, \mathfrak{b})$ we have the following translation table simply writing $(-)^{F}$ and $(-)^{\mathfrak{b}}$ for the action of $F$ and $\mathfrak{b}$ respectively.

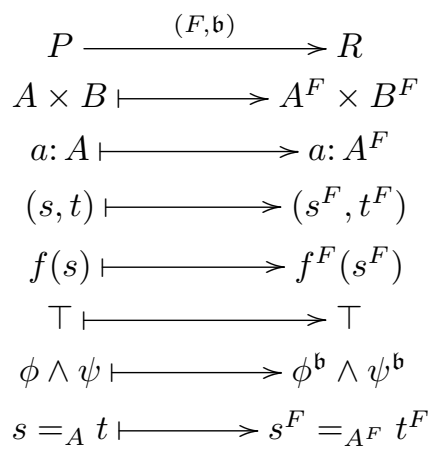

In the same vein, a 2-morphism $\theta:(F, \mathfrak{b}) \rightarrow(G, \mathfrak{c})$ determines a family of terms $\left(x: X^{F} \mid \theta_{X}(x): X^{G}\right)$, as $X$ varies in $\mathcal{C}$, such that, for every term $(a: A \mid t: B)$ in $\mathcal{C}$, the terms $\left(a: A^{F} \mid \theta_{B}\left(t^{F}\right): B^{G}\right)$ and $\left(a: A^{F} \mid t^{G}\left(\theta_{A}(a)\right): B^{G}\right)$ are equal and

$$
a: A^{F} \mid \alpha^{\mathfrak{b}}(a) \vdash \alpha^{\mathfrak{c}}(\theta(a))
$$

for every $A$ in $C$ and $\alpha$ in $P(A)$.

Examples 2.5. (a) Given a theory $\mathcal{T}$ with equality in a first order language $\mathcal{L}$ (say with a single sort), a 1-morphism $(F, \mathfrak{b}): L T \rightarrow S$ from the elementary doctrine $L T: \mathcal{V}^{\mathrm{op}} \longrightarrow \mathbf{I n f S L}$ as in $2.3(\mathrm{~d})$ into $S: \operatorname{Set}^{\mathrm{op}} \longrightarrow$ InfSL, the elementary doctrine in 2.3(a) with $\mathcal{C}=$ Set, determines a model $\mathfrak{M}$ of $\mathcal{T}$ where the set underlying the interpretation is $F\left(x_{1}\right)$. In fact, there is an equivalence between the category $\mathbf{E D}(L T, S)$ and the category of models of $\mathcal{T}$ and $\mathcal{L}$-homomorphisms.

(b) Given a category $\mathcal{C}$ with products and pullbacks, one can consider the two indexed inf-semilattices: that of subobjects $S: C^{\mathrm{op}} \longrightarrow$ InfSL, and the other $\Psi: \mathcal{C}^{\mathrm{op}} \longrightarrow$ InfSL, obtained by the poset reflection of each comma category $C / A$, for $A$ in $C$. The inclusion of the poset $S(A)$ of subobjects over $A$ into the poset reflection of $C / A$ extend to a 1-morphism from $S$ to $\Psi$ which is an equivalence exactly when every morphism in $C$ can be factored as a retraction followed by a monic.

The notion of comprehension is related to the characterization of doctrines of subobjects. Though very general, we shall present that notion in the particular case of an elementary doctrine.

Definition 2.6. Given any elementary doctrine $P: C^{\mathrm{op}} \longrightarrow$ InfSL, and an object $\alpha$ in some $P(A)$, a comprehension of $\alpha$ is a morphism $\{a: A \mid \alpha(a)\}: X \rightarrow$ $A$ in $C$ such that

$$
x: X \mid \top \vdash \alpha(\{a: A \mid \alpha(a)\}(x))
$$


and, for every $f: Z \rightarrow A$ such that

$$
z: Z \mid \top \vdash \alpha(f(z))
$$

there is a unique morphism $f^{\prime}: Z \rightarrow X$ such that $f=\{a: A \mid \alpha(a)\} \circ f^{\prime}{ }^{2}$

Intuitively, the comprehension morphism represents the subsets of elements in the object $A$ obtained by comprehension with the predicate $\alpha$.

In previous works [Maietti and Rosolini, 2013b,a] we said that an elementary doctrine $P$ has comprehensions if every $\alpha$ has a comprehension, and that $P$ has full comprehensions if, moreover, $\alpha \leq \beta$ in $P(A)$ whenever $\{\alpha\}$ factors through $\{\beta\}$.

However to view comprehensions as logical constructors following Jacobs [1999], we need to assume that a choice of comprehensions is available in the doctrine as follows.

An elementary doctrine $P$ has a choice of comprehensions if there is a function $\{-\}$ that assigns a comprehension to every object $\alpha$ in the fibre $P(A)^{3}$, and that $P: C^{\mathrm{op}} \longrightarrow$ InfSL has a choice of full comprehensions if it has a choice comprehensions and, for any $\mathcal{C}$-object $A$, one has $a$ : $A \mid \alpha \vdash \beta$ holds in case there is a factorization

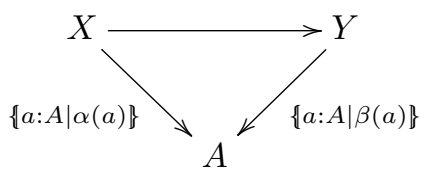

Remark 2.7. The notion of comprehension connects an abstract indexed poset with one of subobjects of the base when this has pullbacks of monomorphisms. We now recall a standard result in the case of interest for us, see e.g. [Jacobs, 1999] where also a very elegant, more abstract view of comprehensions as right adjoint is considered.

Let $P: C^{\mathrm{op}} \longrightarrow$ InfSL be an elementary doctrine, and suppose that $P$ has a choice of comprehensions. Consider the indexed inf-semilattice $S: C^{\mathrm{op}} \longrightarrow$ InfSL of subobjects of the base category $\mathcal{C}$. The function computing comprehensions can be extended to a natural transformation

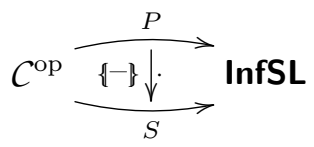

in a unique way. All functors $\{-\}: P(A) \rightarrow S(A)$ are faithful, and are fully faithful exactly when $P$ has full comprehensions.

From now on when we simply say that an elementary doctrine $P$ has comprehensions, or full comprehensions, we assume that it has a choice of comprehensions, or full comprehensions just to be able to use the internal categorical logic.

\footnotetext{
${ }^{2}$ Hence a comprehension is necessarily monic.

${ }^{3}$ It is a function $\{-\} \in \prod_{A \in \mathcal{C}_{0}} \mathcal{C}_{1}^{P(A)_{0}}$.
} 
A special case of comprehensions are the diagonal morphisms and the following definition considers just that possibility.

Definition 2.8. An elementary doctrine $P: C^{\mathrm{op}} \longrightarrow$ InfSL has comprehensive diagonals if every diagonal morphism $\left\langle\operatorname{id}_{A}, \operatorname{id}_{A}\right\rangle: A \rightarrow A \times A$ is a comprehension.

Lemma 2.9. Let $P: C^{o p} \longrightarrow$ InfSL be an elementary doctrine. The following are equivalent:

(i) $P$ has comprehensive diagonals.

(ii) For every object $A$ in $\mathcal{C}$, the diagonal $\left\langle\operatorname{id}_{A}, \operatorname{id}_{A}\right\rangle: A \rightarrow A \times A$ is a comprehension of the corresponding fibered equality $\delta_{A}$.

(iii) For any two morphisms $f, g: A \rightarrow B$ in $\mathcal{C}$, it is

$$
f=g \text { in } C \text { iff } a: A \mid \top \vdash f(a)={ }_{B} g(a) .
$$

It is easy to determine a 2-reflection of elementary doctrines into the full 2 -subcategory of elementary doctrines with comprehensive diagonals once one notices that the condition

$$
a: A \mid \top \vdash f(a)={ }_{B} g(a)
$$

ensures that $P_{f}=P_{g}$, i.e. that, for every $\beta$ in $P(B)$, one has that

$$
a: A \mid \beta(f(a)) \vdash \beta(g(a)) \quad \text { and } \quad a: A \mid \beta(g(a)) \vdash \beta(f(a)) .
$$

So the reflection takes an elementary doctrine $P: C^{\mathrm{op}} \longrightarrow \mathbf{I n f S} \mathbf{L}$ to the elementary doctrine $P_{\mathrm{x}}: \mathcal{C}_{P}^{\mathrm{op}} \longrightarrow \mathbf{I n f S L}$, induced by $P$ on the quotient category $\mathcal{C}_{P}$ of $\mathcal{C}$ with respect to the equivalence relation where $f \sim g$ when

$$
a: A \mid \top \vdash f(a)={ }_{B} g(a) .
$$

We may refer to the doctrine $P_{\mathrm{x}}$ as the extensional reflection of $P$.

Remark 2.10. Note that, when an elementary doctrine $P: C^{\mathrm{op}} \longrightarrow$ InfSL has full comprehensions and comprehensive diagonals, then the base category $\mathcal{C}$ has equalizers. The equalizer of $A \underset{g}{\stackrel{f}{\longrightarrow}} B$ is computed as $\left\{a: A \mid f(a)={ }_{B} g(a)\right\}: E \rightarrow$ A.

Remark 2.11. In the situation considered in 2.7 , the pair $\left(\operatorname{id}_{\mathcal{C}},\{-\}\right)$ is a 1 morphisms in ED

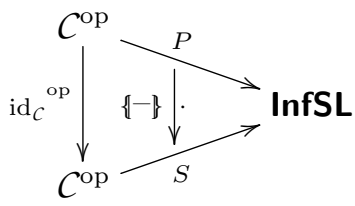


exactly when

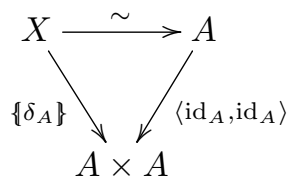

for any objects $A$ in $\mathcal{C}$, i.e. when $P$ has comprehensive diagonals.

Indeed, if $P$ is an elementary doctrine with comprehensions and comprehensive diagonals, then the base category has pullbacks. So $P$ embeds into the indexed poset $S$ of subobjects of $\mathcal{C}$. One can think that comprehensions and comprehsive diagonals force $P$ to "look like" a poset of subobjects of $\mathcal{C}$. Actually, this will be the main situation we shall be interested in the following sections.

With that perspective, we also introduce the notion of existential elementary doctrine from [Lawvere, 1969a], which presents existential quantification as a left adjoint to the substitution functor, see also [Jacobs, 1999].

Definition 2.12. An elementary doctrine $P: C^{\mathrm{op}} \longrightarrow$ InfSL is existential if, for $A_{1}$ and $A_{2}$ in $\mathcal{C}$, for a(ny) projection $\mathrm{pr}_{i}: A_{1} \times A_{2} \rightarrow A_{i}, i=1,2$, the functor $P_{\mathrm{pr}_{i}}: P\left(A_{i}\right) \rightarrow P\left(A_{1} \times A_{2}\right)$ has a left adjoint $\mathbb{B}_{\mathrm{pr}_{i}}$, to which we shall unimaginatively refer as existential, and these left adjoints satisfy the

Beck-Chevalley condition: for any pullback diagram

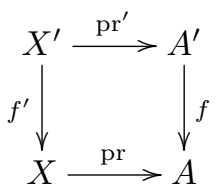

with pr a projection (hence also $\operatorname{pr}^{\prime}$ a projection), for any $\beta$ in $P(X)$, the canonical morphism $\beta_{\mathrm{pr}^{\prime}} P_{f^{\prime}}(\beta) \leq P_{f} \Theta_{\mathrm{pr}}(\beta)$ in $P\left(A^{\prime}\right)$ is iso;

Frobenius reciprocity: for pr: $X \rightarrow A$ a projection, $\alpha$ in $P(A), \beta$ in $P(X)$, the canonical morphism $\mathcal{H}_{\mathrm{pr}}\left(P_{\mathrm{pr}}(\alpha) \wedge_{X} \beta\right) \leq \alpha \wedge_{A} \beta_{\mathrm{pr}}(\beta)$ in $P(A)$ is iso.

Definition 2.13. Let CEED be the 2-full 2-subcategory of ED consisting of existential elementary doctrines with comprehensions whose first components $F$ of 1-morphisms $(F, \mathfrak{b})$ preserve comprehensions and existential adjoints.

Similarly to the case of elementary doctrines, [Jacobs, 1999] shows that the deductive calculus associated to existential elementary doctrines is that of the $\wedge=\exists$-fragment over type theory with a unit type and a binary product type constructor.

Remark 2.14. We recall a result of G.M. Kelly in [Kelly, 1992] which is related to the situation considered in 2.7 when the elementary doctrine $P: C^{\mathrm{op}} \longrightarrow$ InfSL is also existential with full comprehensions; in that case the base category $\mathcal{C}$ has 
a stable proper factorization system. Recall from [Freyd and Kelly, 1972] that a pair $(\mathcal{E}, \mathcal{M})$ of families of morphisms in $\mathcal{C}$ is a factorization system if they satisfy

factorization: every map in $C$ factors as $A \frac{f}{e^{-}{ }_{I} \vec{J}_{m}} B$ where $e$ is in $\mathcal{E}$ and $m$ is in $\mathcal{M}$;

orthogonality: for every commutative diagram $X_{e^{-}}^{f} \int_{g}^{m}$ such that the morphism $e$ in in $\mathcal{E}$ and the morphism $m$ is in $\mathcal{M}$, then there is a unique

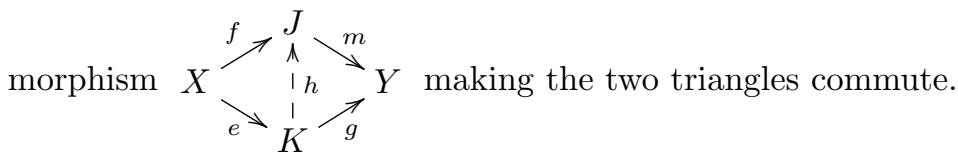

The factorization system is stable if the family $\mathcal{E}$ is closed under pullbacks- $\mathcal{M}$ is automatically closed under pullbacks, thanks to orthogonality-, and it is proper if $\mathcal{M}$ consists of monos and $\mathcal{E}$ consists of epis.

In the factorization system determined on $C$ by the doctrine $P$, the family $\mathcal{M}$ consists of all maps isomorphic to a comprehension and the family $\mathcal{E}$ consists of surjective epis. Given a morphism $f: A \rightarrow B$ in $\mathcal{C}$, a suitable factorization is obtained as follows:

- The mono is $\left\{b: B \mid \exists x: A . f(x)={ }_{B} b\right\}: I \rightarrow B$, the comprehension of the "image in $B$ of the graph of $f$ ".

- Then the first factor is obtained by the universal property of a comprehension since

$$
a: A \mid \top \vdash \exists x: A . f(x)={ }_{B} f(a)
$$

A more general connection between fibrations and not necessarily proper factorization systems than that sketched here is in [Hughes and Jacobs, 2002].

\section{Doctrines with effective quotients}

We saw how in an indexed poset, a comprehension allows to determine a "subset" of definition in the base category for any predicate represented by an object in the fibre. Similarly an equivalence relation in a fibre can determine a quotient in the base category. For that reason, we recall from [Maietti and Rosolini, $2013 \mathrm{~b}, \mathrm{a}]$ the notion of equivalence relation related to a doctrine and the associated notion of effective quotient.

Definition 3.1. Given an elementary doctrine $P: C^{\mathrm{op}} \longrightarrow$ InfSL, an object $A$ in $C$ and an object $\rho$ in $P(A \times A)$, we say that $\rho$ is a $P$-equivalence relation on $A$ if it satisfies 
reflexivity: $a$ : $A, a^{\prime}: A \mid a={ }_{A} a^{\prime} \vdash \rho\left(a, a^{\prime}\right)$;

symmetry: $a: A, a^{\prime}: A \mid \rho\left(a, a^{\prime}\right) \vdash \rho\left(a^{\prime}, a\right)$;

transitivity: $a: A, a^{\prime}: A, a^{\prime \prime}: A \mid \rho\left(a, a^{\prime}\right), \rho\left(a^{\prime}, a^{\prime \prime}\right) \vdash \rho\left(a, a^{\prime \prime}\right)$.

In elementary doctrines as those presented in $2.3, P$-equivalence relations concide with the usual notion for those of the form (a) or (b); more interestingly, in cases like (c) a $\Psi$-equivalence relation is a pseudo-equivalence relation in $\mathcal{S}$ in the sense of [Carboni and Celia Magno, 1982].

For $P: C^{\mathrm{op}} \longrightarrow$ InfSL an elementary doctrine, the object $\left(a: A, a^{\prime}: A \mid a={ }_{A}\right.$ $\left.a^{\prime}\right)$ is a $P$-equivalence relation on $A$. And for a morphism $f: A \rightarrow B$ in $\mathcal{C}$, the functor $P_{f \times f}: P(B \times B) \rightarrow P(A \times A)$ takes a $P$-equivalence relation $\sigma$ on $B$ to a $P$-equivalence relation on $A$. Hence, the $P$-kernel equivalence of $f: A \rightarrow B$, the object ( $\left.a: A, a^{\prime}: A \mid f(a)=_{B} f\left(a^{\prime}\right)\right)$ of $P_{A \times A}$ is a $P$-equivalence relation on $A$. In such a case, one speaks of $\left(a: A, a^{\prime}: A \mid f(a)={ }_{B} f\left(a^{\prime}\right)\right)$ as an effective $P$-equivalence relation.

Definition 3.2. Let $P: C^{\mathrm{op}} \longrightarrow$ InfSL be an elementary doctrine. Let $\rho$ be a $P$-equivalence relation on $A$. A $P$-quotient of $\rho$, or simply a quotient when the doctrine is clear from the context, is a morphism $q: A \rightarrow A / \rho$ in $C$ such that

$$
a ; A, a^{\prime}: A \mid \rho\left(a, a^{\prime}\right) \vdash q(a)={ }_{A / \rho} q\left(a^{\prime}\right)
$$

and, for every morphism $g: A \rightarrow Z$ such that

$$
a ; A, a^{\prime}: A \mid \rho\left(a, a^{\prime}\right) \vdash g(a)={ }_{Z} g\left(a^{\prime}\right),
$$

there is a unique morphism $\bar{g}: A / \rho \rightarrow Z$ such that $g=\bar{g} \circ q$.

We say that such a $P$-quotient is stable if, for every pullback diagram

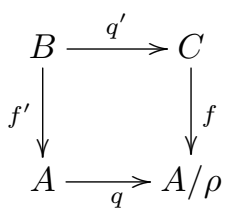

in $\mathcal{C}$, the morphism $q^{\prime}$ is a $P$-quotient.

Definition 3.3. Given an elementary doctrine $P: C^{\mathrm{op}} \longrightarrow$ InfSL and a $P$-equivalence relation $\rho$ on an object $A$ in $\mathcal{C}$, the poset of descent data $\mathcal{D e s} s_{\rho}$ is the sub-poset of $P(A)$ on those $\alpha$ such that

$$
a: A, b: A \mid \alpha(a), \rho(a, b) \vdash \alpha(b) .
$$

Remark 3.4. Given an elementary doctrine $P: C^{\text {op }} \longrightarrow$ InfSL, for $f: A \rightarrow B$ in $C$, let $\chi$ be the $P$-kernel $P_{f \times f}\left(\delta_{B}\right)$. The functor $P_{f}: P(B) \rightarrow P(A)$ applies $P(B)$ into $\mathcal{D e s}_{\chi}$. 
Definition 3.5. Given an elementary doctrine $P: C^{\mathrm{op}} \longrightarrow$ InfSL and a morphism $f: A \rightarrow B$ in $\mathcal{C}$, let $\chi$ be the $P$-kernel $P_{f \times f}\left(\delta_{B}\right)$. The morphism $f$ is descent if the functor $P_{f}: P(B) \rightarrow \mathcal{D} e s_{\chi}$ is full (it is trivially faithful). The morphism $f$ is effective descent if the functor $P_{f}: P(B) \rightarrow \mathcal{D} e s_{\chi}$ is an equivalence (hence an isomorphisms of orders).

Consider the 2-full 2-subcategory QED of ED whose objects are elementary doctrines $P: C^{\mathrm{op}} \longrightarrow$ InfSL with descent quotients of $P$-equivalence relations.

The 1-morphisms are those pairs $(F, \mathfrak{b})$ in ED

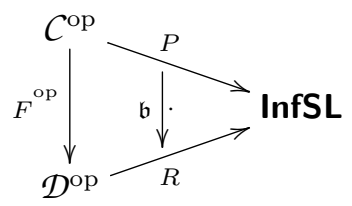

such that $F$ preserves quotients in the sense that, if the morphism $q: A \rightarrow$ $C$ in $C$ is a quotient of a $P$-equivalence relation $\rho$ on $A$, then the morphism $F q: F A \rightarrow F C$ in $\mathcal{D}$ is a quotient of the $R$-equivalence relation $R_{\left\langle F\left(\mathrm{pr}_{1}\right), F\left(\mathrm{pr}_{2}\right)\right\rangle}\left(\mathfrak{b}_{A \times A}(\rho)\right)$ on $F A$.

In [Maietti and Rosolini, 2013a] we proved that it is possible to add freely quotients of equivalence relations to an elementary doctrine. For an elementary doctrine $P: C^{\mathrm{op}} \longrightarrow$ InfSL, the quotient completion of $P$ consists of a category $Q_{P}$ of "quotients in $P$ ", defined as follows:

an object of $Q_{P}$ is a pair $(A, \rho)$ such that $\rho$ is a $P$-equivalence relation on $A$;

a morphism $f:(A, \rho) \rightarrow(B, \sigma)$ is a morphism $f: A \rightarrow B$ in $C$ such that

$$
a: A, b: A \mid \rho(a, b) \vdash \sigma(f(a), f(b)) .
$$

Composition is given by that of $\mathcal{C}$, identities are represented by identities of $\mathcal{C}$.

The indexed inf-semilattice $\widehat{P}: Q_{P}^{\mathrm{op}} \longrightarrow$ InfSL on $Q_{P}$ is the categories of descent data: on an object $(A, \rho)$ it is defined as

$$
\widehat{P}(A, \rho):=\mathcal{D e s}_{\rho}
$$

where the order of descent data $\mathcal{D e s}_{\rho}$ is the sub-order of $P(A)$ on those $\alpha$ such that

$$
a: A, b: A \mid \alpha(a), \rho(a, b) \vdash \alpha(b) .
$$

There is a 1-morphism $(J, j): P \rightarrow \widehat{P}$ in ED where $J: C^{\text {op }} \longrightarrow \mathcal{R}_{P}$ sends an object $A$ in $C$ to $\left(A, \delta_{A}\right)$ and a morphism $f: A \rightarrow B$ to $f:\left(A, \delta_{A}\right) \rightarrow\left(B, \delta_{B}\right)$ since $\delta_{A} \leq_{A \times A} P_{f \times f}\left(\delta_{B}\right)$, and $j_{A}: P(A) \rightarrow \widehat{P}\left(A, \delta_{A}\right)$ is the identity since

$$
\widehat{P}\left(A, \delta_{A}\right)=\mathcal{D e s}_{\delta_{A}}=P(A) .
$$

It is immediate to see that $J$ is full and faithful and that $(J, j)$ is a change of base. 
Theorem 3.6. The indexed inf-semilattice $\widehat{P}: Q_{P}^{o p} \longrightarrow$ InfSL is an elementary doctrine with effective quotients, and it is the free such on $P$ in the sense that, for every elementary doctrine $P: C^{o p} \longrightarrow$ InfSL, pre-composition with the 1morphism

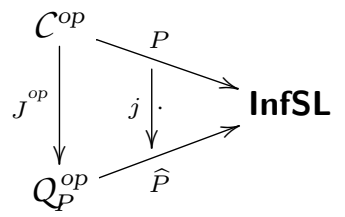

in ED induces an essential equivalence of categories

$$
\text { -。 }(J, j): \mathbf{Q E D}(\widehat{P}, Z) \equiv \mathbf{E D}(P, Z)
$$

for every $Z$ in QED.

As noticed in [Maietti and Rosolini, 2013a] the completion with quotients commute with other fibrational structure.

Proposition 3.7. Let $P: C^{o p} \longrightarrow$ InfSL be an elementary doctrine and let $\widehat{P}: Q_{P}^{o p} \longrightarrow$ InfSL be its quotient completion. Then the following properties hold.

(i) If $P$ has comprehensions, then $\widehat{P}$ has comprehensions.

(ii) If $P$ has full comprehensions, then $\widehat{P}$ has full comprehensions and effective quotients are stable.

(iii) If $P$ is existential, then $\widehat{P}$ is existential.

Note that comprehension is not preserved by the embedding morphism $(J, j): P \rightarrow \widehat{P}$ so swiftly (see [Maietti and Rosolini, 2013b,a]).

Proposition 3.8. Let $P: C^{o p} \longrightarrow$ InfSL be an elementary doctrine with comprehensive diagonals. If $P$ has full comprehensions, then $(J, j)$ preserves them.

Moreover, the property of comprehensive diagonals is not necessarily inherited by the free completion $\widehat{P}$.

Therefore, if one wants to construct from an elementary doctrine $P: C^{\mathrm{op}} \longrightarrow$ InfSL, in a free way, an indexed poset with effective quotients which looks as much as possible like the indexed poset of subobjects of the base category-i.e. the indexed poset has full comprehension and comprehensive diagonals-, one must go through a few steps:

(a) make sure that $P$ has full comprehensions and comprehensive diagonals;

(b) add effective quotients;

(c) force comprehensive diagonals. 
As for step (a), in case $P$ fails to have comprehensions or comprehensive diagonals, these can be added freely, see e.g. [Maietti and Rosolini, 2013a].

Step (b) is just an application of 3.6.

Step (c) is accomplished by the construction after 2.9 once one checks that

$\widehat{P}_{\mathrm{x}}$ has effective quotients. Moreover, full comprehensions are inherited by $\widehat{P}_{\mathrm{x}}$, as well as existential quantifiers, if $P$ has them.

In the following sections, we shall analyze a situation which arises when

$$
\widehat{P}_{\mathrm{x}}:\left(\left(Q_{P}\right)_{\widehat{P}}\right)^{\mathrm{op}} \longrightarrow \mathbf{I n f S L}
$$

is the fibration of subobjects of its base category. We refer to such a doctrine $\widehat{P}_{\mathrm{x}}$ as the elementary quotient completion of $P$.

\section{The rule of unique choice}

In this section we are going to show when an elementary quotient completion of an elementary doctrine happens to be an exact category by making use of a choice principle.

As a first step, it is useful to recall the definition of the exact completion of an existential elementary doctrine introduced in [Maietti and Rosolini, 2015]; we refer the reader to loc.cit. for the details, pointing out that there are many sources in the literature which discuss various different presentations of the completion, e.g. [Kelly, 1992, Carboni, 1982, Carboni and Walters, 1987, Freyd and Scedrov, 1991, Jacobs, 1999, Hughes and Jacobs, 2002].

For the purpose of the following presentation, it is convenient to introduce a preliminary concept which requires precisely the notion of existential elementary doctrine.

Definition 4.1. Let $P: C^{\mathrm{op}} \longrightarrow$ InfSL be an elementary existential doctrine. Let $\phi$ be an object in $P(A \times B)$. One says that $\phi$ is functional from $A$ to $B$ if the following conditions hold:

$\phi$ is single-valued: $\quad a: A, b: B, b^{\prime}: B \mid \phi(a, b), \phi\left(a, b^{\prime}\right) \vdash b={ }_{B} b^{\prime}$;

$\phi$ is total: $\quad a: A \mid \top \vdash \exists b: B . \phi(a, b)$.

Definition 4.2. An elementary existential doctrine $P: C^{\mathrm{op}} \longrightarrow$ InfSL satisfies the rule of unique choice if, for pair of objects $A$ and $B$, and every functional object $\phi$ from $A$ to $B$ there is a morphism $f: A \rightarrow B$ in $C$ such that

$$
a: A \mid \top \vdash \phi(a, f(a)) .
$$

Example 4.3. The existential elementary doctrine $S: S e t^{\text {op }} \longrightarrow$ InfSL of subsets satisfies the rule of unique choice.

The reader may appreciate a very close relationship between the concept introduced in 4.2 and that of a relation which is a function. Indeed, like for that 
one, one can introduce a notion of functional composition as follows: for $\phi$ functional from $A$ to $B$ and $\psi$ functional from $B$ to $C$, it is

$$
\exists b: B \cdot[\phi(a, b) \wedge \psi(b, c)]
$$

with $a: A$ and $c: C$.

It should now appear clear that, for a given existential elementary doctrine $P: C^{\mathrm{op}} \longrightarrow$ InfSL, there is a category $\mathcal{F}_{P}$ of functional morphisms on it whose objects are the objects of $C$ and where a morphism $\phi: A \rightarrow B$ is a functional object from $A$ to $B$. Composition is functional composition; the identity morphism on $A$ is $\left(a: A, a^{\prime}: A \mid a={ }_{A} a^{\prime}\right)$ which is clearly functional from $A$ to $A$, see [Freyd and Scedrov, 1991].

There is a natural functor $K: C \longrightarrow \mathcal{F}_{P}$ which is the identity on the objects and maps $f: A \rightarrow B$ in $C$ to the functional object $\left(a: A, b: B \mid f(a)={ }_{B} b\right)$ in $P(A \times B)$. The functor $K$ is faithful exactly when $P$ has comprehensive diagonals.

As $K$ suggests, the data for $\mathcal{F}_{P}$ appear as superimposed on $\mathcal{C}$. So, in order to avoid confusion, from now on we shall mark morphisms in $\mathcal{F}_{P}$ with an asterisk as follows $\phi: A \rightarrow_{*} B$.

Since the initial datum is an existential elementary doctrine, one expects to determine a similar structure on $\mathcal{F}_{P}$. Indeed, the similarity with the set-theoretic situation continues as one defines the indexed poset $P^{+}$as $P^{+}(A):=P(A)$ and, for a morphism $\phi: A \rightarrow_{*} B$ in $\mathcal{F}_{P}$, a homomorphism of inf-semilattices

$$
P^{+}(\phi):=[(y: B \mid \alpha(y)) \mapsto(x: A \mid \exists y: B .(\phi(x, y) \wedge \alpha(y)))] .
$$

The following result follows directly from the definition of the rule of unique choice.

Proposition 4.4. Let $P: C^{o p} \longrightarrow$ InfSL be an existential elementary doctrine. $P$ satisfies the rule of unique choice if and only if its extensional reflection $P_{\mathrm{x}}: C_{P}^{o p} \longrightarrow$ InfSL does.

Proof. It is obvious since the fibres of $P_{\mathrm{x}}$ are the same as those of $P$ and there is simply an existence condition on the morphism $f$ in the rule of unique choice.

Proposition 4.5. The indexed inf-semilattice $P^{+}: \mathcal{F}_{P}^{o p} \longrightarrow$ InfSL is an existential elementary doctrine with comprehensive diagonals which satisfies the rule of unique choice. Moreover

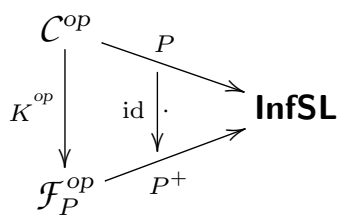

is a 1-morphism in EED . 
Proof. It is a direct computation after one notices that binary products are induced by $K$, so the product of $A$ and $B$ is

$$
A<\frac{K\left(\mathrm{pr}_{1}\right)}{} A \times B \stackrel{K\left(\mathrm{pr}_{2}\right)}{\longrightarrow} B .
$$

Remark 4.6. In fact, $(K$, id) is a 2-reflection of EED into its full 2-subcategory on the existential elementary doctrines with comprehensive diagonals which satisfies the rule of unique choice. So $(K$, id $)$ is an equivalence exactly when $P: C^{\mathrm{op}} \longrightarrow$ InfSL has comprehensive diagonals and satisfies the rule of unique choice.

In fact, $K$ is full if and only if $P$ satisfies the rule of unique choice, and $K$ is faithful if and only if $P$ has comprehensive diagonals.

Note that one obtains the exact completion $\mathcal{E}_{P}$ of an elementary existential doctrine $P$ in [Maietti and Rosolini, 2015] as the doctrine $\mathcal{F}_{\widehat{P}}$.

Remark 4.7. It is easy to recognize that examples of exact completion for the definition given here is the exact completion $X_{\text {ex/reg }}$ of a regular category $\mathcal{X}$, see [Freyd and Scedrov, 1991, Carboni, 1995, Carboni and Vitale, 1998], by taking the subobject fibration of $X$ as $P$.

Other examples come from theories apt to formalize constructive mathematics: the category of total setoids à la Bishop and functional relations based on the intensional type theory of the Minimalist Foundation in [Maietti, 2009], or the category of total setoids à la Bishop and functional relations based on the Calculus of Constructions [Coquand, 1990], which forms a topos as mentioned in [Barthe et al., 2003].

Remark 4.8. Note that an existential elementary doctrine $P: C^{\mathrm{op}} \longrightarrow$ InfSL with comprehensions has unique choice in the sense of [Jacobs, 1999] if and only if it satisfies the rule of unique choice. So, it follows from 4.4.4 and 4.9.4 of loc.cit. that the following are equivalent.

(i) $C$ is a regular category and $P$ is the doctrine of its subobject.

(ii) $P$ has full comprehensions, comprehensive diagonals and satisfies the rule of unique choice.

Theorem 4.9. Let $P: C^{o p} \longrightarrow$ InfSL be an existential elementary doctrine with stable effective quotients. The following are equivalent.

(i) $C$ is an exact category and $P$ is the doctrine of its subobjects.

(ii) $P$ has full comprehensions, comprehensive diagonals and satisfies the rule of unique choice. 
Proof. It follows from 4.8 because, for a subobject fibration $S: C^{o p} \longrightarrow \operatorname{InfSL}$, an $S$-equivalence relation is precisely an equivalence relation in $C$ and an exact category is a regular category with effective coequalizers of equivalence relations.

Recall from [Maietti and Rosolini, 2015] the following rule of choice.

Definition 4.10. Given an existential elementary doctrine $P: C^{\mathrm{op}} \longrightarrow$ InfSL we say that $P$ satisfies the rule of choice if, for every $\phi \in P(A \times B)$ such that

$$
a: A \mid \top \vdash \exists b: B . \phi(a, b),
$$

there is a morphism $f: A \rightarrow B$ in $C$ such that

$$
a: A \mid \top \vdash \phi(a, f(a)) .
$$

Clearly the rule of choice is stronger than the rule of unique choice since the relations employed in the rule of unique choice are particular instances of those which appear in the rule of choice.

Proposition 4.11. Let $P: C^{o p} \longrightarrow$ InfSL be an existential elementary doctrine with comprehensions. Then $P$ satisfies the rule of choice if and only if its quotient completion $\widehat{P}: Q_{P}^{o p} \longrightarrow$ InfSL satisfies the rule of unique choice.

Proof. $(\Rightarrow)$ Immediate.

$(\Leftarrow)$ Suppose $\phi \in P(A \times B)$ is such that

$$
a: A \mid \top \vdash \exists b: B . \phi(a, b) .
$$

Consider the comprehension $\ell:=\{a: A, b: B \mid \phi(a, b)\}: X \rightarrow A \times B$ and consider the object

$$
\left(X,\left(x: X, x^{\prime}: X \mid \operatorname{pr}_{1}(\ell(x))={ }_{A} \operatorname{pr}_{1}\left(\ell\left(x^{\prime}\right)\right)\right)\right)
$$

in $Q_{P}$. It is immediate to check that $\left(a: A, x: X \mid a={ }_{A} \operatorname{pr}_{1}(\ell(x))\right)$ is in

$$
\widehat{P}\left(\left(A,\left(a: A, a^{\prime}: A \mid a={ }_{A} a^{\prime}\right)\right) \times\left(X,\left(x: X, x^{\prime}: X \mid \operatorname{pr}_{1}(\ell(x))={ }_{A} \operatorname{pr}_{1}\left(\ell\left(x^{\prime}\right)\right)\right)\right)\right)
$$

since it is just

$a: A, a^{\prime}: A, x: X, x^{\prime}: X \mid a={ }_{A} a^{\prime}, \operatorname{pr}_{1}(\ell(x))={ }_{A} \operatorname{pr}_{1}\left(\ell\left(x^{\prime}\right)\right), a={ }_{A} \operatorname{pr}_{1}(\ell(x)) \vdash a^{\prime}={ }_{A} \operatorname{pr}_{1}\left(\ell\left(x^{\prime}\right)\right)$.

It is also easy to prove that $\left(a: A, x: X \mid a={ }_{A} \operatorname{pr}_{1}(\ell(x))\right)$ is functional from $\left(A,\left(a: A, a^{\prime}: A \mid a={ }_{A} a^{\prime}\right)\right)$ to $\left(X,\left(x: X, x^{\prime}: X \mid \operatorname{pr}_{1}(\ell(x))={ }_{A} \operatorname{pr}_{1}\left(\ell\left(x^{\prime}\right)\right)\right)\right)$ since the existential quantifiers of $\widehat{P}$ are those of $P$ as shown in [Maietti and Rosolini, 2013b].

Thanks to the assumption that $\widehat{P}$ satisfies the rule of unique choice, there is $f: A \rightarrow X$ such that

$$
a: A \mid \top \vdash a={ }_{A} \operatorname{pr}_{1}(\ell(f(a))) .
$$

Hence the morphism $\operatorname{pr}_{2} \circ \ell \circ f: A \rightarrow B$ is such that $a: A \mid \top \vdash \phi\left(a, \operatorname{pr}_{2}(\ell(f(a)))\right)$. 


\section{The rule of choice and exact completions}

Lemma 5.1. Let $P: C^{o p} \longrightarrow$ InfSL be an existential elementary doctrine, and consider the 1-morphism

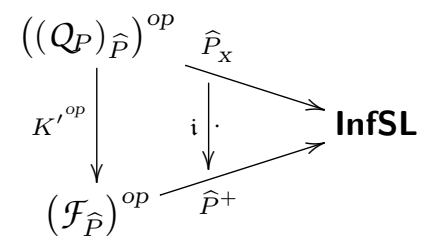

induced by the 1-morphism (K,id): $\widehat{P} \rightarrow \mathcal{F}_{\widehat{P}}$. The functor $K^{\prime}$ is full (hence iso) if and only if $P$ satisfies the rule of choice.

Proof. It follows from 4.4 and 4.11 .

Theorem 5.2. Let $P: C^{o p} \longrightarrow$ InfSL be an existential elementary doctrine with comprehensive diagonals, full comprehensions and let $\widehat{P}_{\mathrm{x}}:\left(\left(Q_{P}\right)_{\widehat{P}}\right)^{o p} \longrightarrow$ InfSL be its elementary quotient completion. The following are equivalent.

(i) the base category $\left(Q_{P}\right)_{\widehat{P}}$ of the elementary quotient completion of $P$ is an exact category and $\widehat{P}_{\mathrm{x}}$ is its subobject doctrine.

(ii) $P$ satisfies the rule of choice.

(iii) the base category $\left(Q_{P}\right)_{\widehat{P}}$ of the elementary quotient completion of $P$ is equivalent to the exact completion $\mathcal{E}_{P} \equiv \mathcal{F}_{\widehat{P}}$ of $P$ and $\widehat{P}_{\mathrm{x}}$ is a subobject doctrine.

Proof. It follows from 5.1 and 4.9 .

Remark 5.3. Note that proposition 4.11 and theorem 5.2 hold also if $P$ has just weak full comprehensions.

Examples 5.4. The setoid model over Martin-Löf's type theory in [Maietti and Rosolini, 2013b] and the elementary quotient completion of the doctrine of a lex category (with a choice of its structure) provide examples of elementary quotient completions which are exact since the rule of unique choice holds in the logic of the quotient model.

\section{The axiom of unique choice in an elementary quotient completion}

Proposition 4.11 continues to hold if we replace the rule of choice with the axiom of choice and the rule of unique choice with the axiom of unique choice respectively, provided that the doctrine $P$ has implications and universal quantifications and its base category is closed under (weak) function spaces. We recall the basic notions to state that result. 
Definition 6.1. An elementary doctrine $P: C^{\mathrm{op}} \longrightarrow$ InfSL is implicational if, for every object $A$ in $\mathcal{C}$, every $\alpha$ in $P(A)$, the functor $\alpha \wedge-: P(A) \rightarrow P(A)$ has a right adjoint $\alpha \Rightarrow-: P(A) \rightarrow P(A)$.

Definition 6.2. An elementary doctrine $P: C^{\mathrm{op}} \longrightarrow$ InfSL is universal if, for $A_{1}$ and $A_{2}$ in $C$ and a(ny) projection $\operatorname{pr}_{i}: A_{1} \times A_{2} \rightarrow A_{i}, i=1,2$, the functor $P_{\mathrm{pr}_{i}}: P\left(A_{i}\right) \rightarrow P\left(A_{1} \times A_{2}\right)$ has a right adjoint $V_{\mathrm{pr}_{i}}$, and these satisfy the BeckChevalley condition: for any pullback diagram

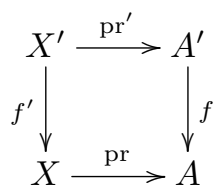

with pr a projection (hence also $\mathrm{pr}^{\prime}$ a projection), for any $\beta$ in $P(X)$, the canonical arrow $P_{f} V_{\mathrm{pr}}(\beta) \leq{V_{\mathrm{pr}}}_{f_{f^{\prime}}}(\beta)$ in $P\left(A^{\prime}\right)$ is iso.

Definition 6.3. An elementary existential implicational and universal doctrine $P: C^{\mathrm{op}} \longrightarrow$ InfSL with a cartesian closed base satisfies the axiom of unique choice, (AUC) for short, if

$$
(\forall x: A . \exists ! y: B . \phi(x, y)) \rightarrow\left(\exists f: B^{A} . \forall x: A . \phi(x, f(x))\right)
$$

holds in $P$.

As already noticed in [Maietti and Rosolini, 2013b] the completion with quotients commute also with the fibrational structure described above.

Proposition 6.4. Let $P: C^{o p} \longrightarrow$ InfSL be an elementary doctrine with full comprehensions and let $\widehat{P}: Q_{P}^{o p} \longrightarrow \mathbf{I n f S L}$ be its quotient completion. Then the following properties hold.

(i) If $C$ is cartesian closed then $Q_{P}$ is cartesian closed.

(ii) If $P$ is implicational, then $\widehat{P}$ is implicational.

(iii) If $P$ is universal, then $\widehat{P}$ is universal.

Moreover, the 1-arrow $(J, j): P \rightarrow \widehat{P}$ preserves the implicational and universal structure of $P$.

Finally, we have:

Proposition 6.5. Let $P: C^{o p} \longrightarrow$ InfSL be an elementary existential implicational and universal doctrine with a cartesian closed base and full comprehensions. Then $P$ satisfies the axiom of choice if and only if its quotient completion $\widehat{P}: Q_{P}^{o p} \longrightarrow$ InfSL satisfies the axiom of unique choice. 
Proof. The proof is similar to that of proposition 4.11 .

$\Leftrightarrow)$ Immediate by the definition of right adjoints and implications in $\widehat{P}$.

$(\Leftarrow)$ Suppose $\phi \in P(A \times B)$ is $P$-relation. Consider the comprehension $\ell:=\{a: A, b: B \mid \phi(a, b)\}: X \rightarrow A \times B$ and the relation

$$
\left(a: A, x: X \mid a={ }_{A} \operatorname{pr}_{1}(\ell(x))\right)
$$

which is in

$$
\widehat{P}\left(\left(A,\left(a: A, a^{\prime}: A \mid a={ }_{A} a^{\prime}\right)\right) \times\left(X,\left(x: X, x^{\prime}: X \mid \operatorname{pr}_{1}(\ell(x))={ }_{A} \operatorname{pr}_{1}\left(\ell\left(x^{\prime}\right)\right)\right)\right)\right)
$$

Then, (AUC) holds in $\widehat{P}$ for that $\widehat{P}$-relation and that yields that (AC) holds for $\phi$ in $P$ since the existential quantifiers, the universal quantifiers and implications of $\widehat{P}$ are defined in terms of those of $P$ as shown in [Maietti and Rosolini, 2013b].

Remark 6.6. Observe that, as noticed in [Maietti, 2005], the subobject doctrine of a locally cartesian closed regular category is implicational, universal and validates the axiom of unique choice.

Remark 6.7. Note that proposition 6.4 and theorem 6.5 hold also if $P$ has just weak full comprehensions and its base is only weakly cartesian closed.

An example of this structure is the setoid model over Martin-Löf's type theory of example 5.4. A discussion on the validity of choice principles in such a structure can be found in [Martin-Löf, 2006]. Proposition 6.5 (with weak full

comprehensions and a weak cartesian closed base) gives an abstract account of what happens in this example.

\section{References}

G. Barthe, V. Capretta, and O. Pons. Setoids in type theory. J. Funct. Programming, 13(2):261-293, 2003.

A. Carboni. Analysis non-standard e topos. Rend. Istit. Mat. Univ. Trieste, 14 (1-2):1-16, 1982. ISSN 0049-4704.

A. Carboni. Some free constructions in realizability and proof theory. J. Pure Appl. Algebra, 103:117-148, 1995.

A. Carboni and R. Celia Magno. The free exact category on a left exact one. J. Aust. Math. Soc., 33(A):295-301, 1982.

A. Carboni and E.M. Vitale. Regular and exact completions. J. Pure Appl. Algebra, 125:79-117, 1998.

A. Carboni and R.F.C. Walters. Cartesian bicategories, I. J. Pure Appl. Algebra, 49:11-32, 1987. 
T. Coquand. Metamathematical investigation of a calculus of constructions. In P. Odifreddi, editor, Logic in Computer Science, pages 91-122. Academic Press, 1990.

P.J. Freyd and G.M. Kelly. Categories of continuous functors I. J. Pure Appl. Algebra, 2:169-191, 1972.

P.J. Freyd and A. Scedrov. Categories Allegories. North Holland Publishing Company, 1991.

M. Grandis. Weak subobjects and the epi-monic completion of a category. $J$. Pure Appl. Algebra, 154(1-3):193-212, 2000.

J. Hughes and B. Jacobs. Factorization systems and fibrations: Toward a fibred Birkhoff variety theorem. Electron. Notes Theor. Comput. Sci., 11:\#, 2002. URL http://www.elsevier.nl/locate/entcs/volume6.html.

J.M.E. Hyland. The effective topos. In A.S. Troelstra and D. van Dalen, editors, The L.E.J. Brouwer Centenary Symposium, pages 165-216. North Holland Publishing Company, 1982.

J.M.E. Hyland, P.T. Johnstone, and A.M. Pitts. Tripos Theory. Math. Proc. Camb. Phil. Soc., 88:205-232, 1980.

B. Jacobs. Categorical Logic and Type Theory. North Holland Publishing Company, 1999.

G.M. Kelly. A note on relations relative to a factorization system. In A. Carboni, M.C. Pedicchio, and G. Rosolini, editors, Category Theory '90, volume 1488 of Lecture Notes in Math., pages 249-261. Springer-Verlag, Como, 1992.

J. Lambek and P.J. Scott. Introduction to Higher Order Categorical Logic. Cambridge University Press, 1986.

F. W. Lawvere. Adjointness in foundations. Dialectica, 23:281-296, 1969a.

F.W. Lawvere. Diagonal arguments and cartesian closed categories. In Category Theory, Homology Theory and their Applications, II (Battelle Institute Conference, Seattle, Wash., 1968, Vol. Two), pages 134-145. Springer, 1969b.

F.W. Lawvere. Equality in hyperdoctrines and comprehension schema as an adjoint functor. In A. Heller, editor, Proc. New York Symposium on Application of Categorical Algebra, pages 1-14. Amer.Math.Soc., 1970.

F.W. Lawvere and R. Rosebrugh. Sets for Mathematics. Cambridge University Press, 2003.

Maria Emilia Maietti. Joyal's arithmetic universe as list-arithmetic pretopos. Theory Appl. Categ., 3:No. 24, 39-83, 2010. 
M.E. Maietti. Modular correspondence between dependent type theories and categorical universes including pretopoi and topoi. Math. Structures Comput. Sci., 15(6):1089-1149, 2005.

M.E. Maietti. A minimalist two-level foundation for constructive mathematics. Ann. Pure Appl. Logic, 160(3):319-354, 2009.

M.E. Maietti and G. Rosolini. Elementary quotient completion. Theory Appl. Categ., 27:445-463, 2013a.

M.E. Maietti and G. Rosolini. Quotient completion for the foundation of constructive mathematics. Log. Univers., pages 371-402, 2013b.

M.E. Maietti and G. Rosolini. Unifying exact completions. Appl. Categ. Structures, 23:43-52, 2015.

M. Makkai and G. Reyes. First Order Categorical Logic, volume 611 of Lecture Notes in Math. Springer-Verlag, 1977.

P. Martin-Löf. 100 years of Zermelo's axiom of choice: what was the problem with it? The Computer Journal, 49(3):10-37, 2006.

P. Taylor. Practical Foundations of Mathematics. Cambridge University Press, 1999. URL ftp://ftp.dcs.qmw.ac.uk/pub/lfp/pt/book-contents.

J. van Oosten. Realizability: An Introduction to its Categorical Side, volume 152. North Holland Publishing Company, 2008. 